\title{
LA PREVISIÓN SOCIAL COMPLEMENTARIA EN EL MARCO DE LAS RELACIONES LABORALES: EL CASO DE MAIER
}

\author{
Rosa María Ahumada Carazo \\ Profesora del Departamento Economía Financiera II UPV/EHU \\ ENEa Ispizua Dorna
}

Licenciada en Derecho e investigadora en el Departamento de Derecho de Empresa UPV/EHU

DOI: 10.1387/lan-harremanak.15399

\section{ABSTRACT}

A lo largo de los últimos años se han realizado numerosas reformas que afectan al sistema de la Seguridad Social, tanto en el ámbito nacional como internacional. Observando el panorama nacional, se han adoptado una serie de medidas, siguiendo las recomendaciones de la OCDE y la Unión Europea. El efecto de esas medidas ya se está empezando a notar en la caída en la tasas de sustitución de la renta de pasivo sobre la de activo, con la consiguiente necesidad de complementar la pensión pública. Aunque son muchos los que defienden la cobertura de ese déficit a través de la previsión social complementaria individual, en este trabajo mostramos la idoneidad de fomentar la previsión social complementaria dentro del marco de las relaciones laborales. Esto se viene haciendo tradicionalmente en algunos paises europeos. En el trabajo se analizan las caracteristicas del sistema complementario de previsión social de una empresa del País Vasco, asi como la opinión de su personal sobre el mismo a través de una encuesta. Finalmente, se presentan las conclusiones del marco teórico y práctico asi como una relación de propuestas.

Palabras clave: Seguridad Social, protección social complementaria, OCDE, Unión Europea, MAIER. 
Azken urteotan Gizarte Segurantzaren gainean hainbat erreforma burutu dira, bai estatu mailan zein nazioarte mailan. Estatu mailan, ELGA-k eta Europar Batasunak emandako gomendioak jarraitu dituzte erreformak burutzerako orduan. Hartutako neurrien ondorioak nabaritzen hasi dira, batez ere ordezkapen tasaren jaitsieran. Ondorioz, errenta publikoa osatzeko premia ikusten da. Nabiz eta petsona asko diren beharrizan hau gizarte babes osagarri indibidualaren bidez betetzea defendatzen dutenak, lan honen bidez gizate babes osagarri kolektiboaren egokitasuna aztertuko dugu, lan harremanen barnean. Tradizionalki, zenbait europar herrialdetan egin izan da. Lan honetan, gizarte babes osagarriaren ezaugarriak aztertzen dira Euskara Autonomi-Erkidegoko enpresa batean eta baita enpresa horretako lankideen iritzia sistemarekiko inkesta baten bidez. Azkenik, ondorio teoriko eta praktikoak aurkezten dira, enpreari emandako proposamenak azaleratuz.

Hitz gakoak: Gizarte Segurantza, gizarte babes osagarria, ELGA, Europar Batasuna, MAIER.

During the last years many reforms have been made which directly affect the social security system, both in the national and intenational fields. Taking notice of the national scene, some measures have been taken following OECD and European Union recomendations. The effect caused by those measures has started to be noticeable in the fall of the rate of replacement of the passive income over the active income, which consequently creates the need of complementing the state pension. Although there are many who uphold the idea of covering that shortage through the individual supplementary pension, in this work we put forth the suitability of promoting the supplementary individual pension within the labor relations frame. This is being done in some European countries. In this work, the characteristics of the individual supplementary pension of a company in the Basque Country are analyzed, also the opinion of the staff about it through an inquiry. Finally, conclusions are presented of the legal and practical framework together with a list of proposals.

Key Words: Social security, complementary social protection, OECD, European Union, MAIER. 


\section{Introducción}

En los últimos años, se están observando numerosas reformas que afectan al sistema de protección social, tanto en el ámbito nacional como internacional. Observando el panorama nacional, se han adoptado una serie de medidas para hacer frente al envejecimiento de la población, siguiendo las recomendaciones de la OCDE y la Unión Europea. Entre ellas se cita el fomento de la previsión social complementaria. En el contexto de las relaciones laborales, existen empresas que hace tiempo pusieron en marcha un sistema de previsión social complementario. Este es el caso de las Cooperativas de Mondragón Corporación, y por ejemplo de Maier S.Coop., en la que nos centraremos. Antes de entrar en el análisis del sistema de previsión social de esta empresa, creemos necesario realizar una breve contextualización sobre la protección social.

\subsection{El modelo de protección social: estructura y objeto de protección}

La Constitución Española no contempla la noción de protección social como tal, pero si las de la Seguridad Social, Asistencia Social o Sanidad. Sin embargo, el Sistema Europeo de Estadísticas Integradas de Protección Social $^{1}$, SEEPROS, ha definido la protección social como "todas aquellas intervenciones públicas o privadas destinadas a aligerar la carga que representa para los hogares y particulares una serie de riesgos o necesidades, previamente determinados, siempre que no exista una contrapartida simultánea o recíproca por parte del beneficiario" (López, 2011). Dentro de esta definición entrarían las prestaciones por enfermedad, atención sanitaria, invalidez, vejez, supervivencia, familia, desempleo, vivienda y exclusión social, tanto de origen público como privado.

Adicionalmente, la doctrina ha ido perfilando la definición de protección social. Así, Borrajo (Alarcón, 1996), apuntó los cuatro elementos que la pro-

\footnotetext{
${ }^{1}$ Ver http://europa.eu/legislation_summaries/employment_and_social_policy/situation_in_ europe/c10141_es.htm
} 
tección social debería tener, a saber: la Seguridad Social pública, con carácter preferentemente contributivo; la Asistencia Social pública, con carácter preferentemente no contributivo; los Servicios Sociales, gestionados por las Comunidades Autónomas o por Entidades Locales y, por último, el Servicio de la Salud.

Por otro lado, para Hurtado González, dentro de la protección social se encuentran la Seguridad Social, la Asistencia Social y las demás prestaciones públicas que se han calificado como política social o bienestar social. Este mismo autor aporta las posibles relaciones entre protección social y Seguridad Social: que la Seguridad Social y la protección social sean equivalentes; que la Seguridad Social sea más amplia que la protección social y que la $\mathrm{Se}$ guridad Social se encuentre dentro de la protección social. Esta última posibilidad cree que es la más lógica (Alarcón, 1996). Asimismo, Alarcón Caracuel hace una doble distinción dentro del mismo concepto y lo clasifica en Públicos y Privados. Entre los Públicos u obligatorios entrarían el Sistema Nacional de Salud, el sistema de Seguridad Social ${ }^{2}$, la asistencia social y otras formas de protección social pública. Por su lado, en los Privados y libres estarían la seguridad social complementaria, los Planes y Fondos de Pensiones y otras formas de protección privada. Este mismo autor afirma que, a la hora de diferenciar la Protección Social de la Seguridad Social, hay que tener en cuenta que mientras el primero puede tener carácter privado, el segundo siempre es público.

En la Unión Europea existe una gran variedad de sistemas de protección social en función del país. En términos generales, el sistema global de pensiones se puede reestructurar en tres niveles como se indicaba en el Libro Verde sobre los Sistemas Complementarios de Pensiones en el mercado único (1997). Todo ello se recoge en la figura 1.

2 Incluiría las prestaciones económicas tanto contributivas como no contributivas; y prestaciones en especie, que serían los servicios sociales. 
Figura 1

Sistema de protección social
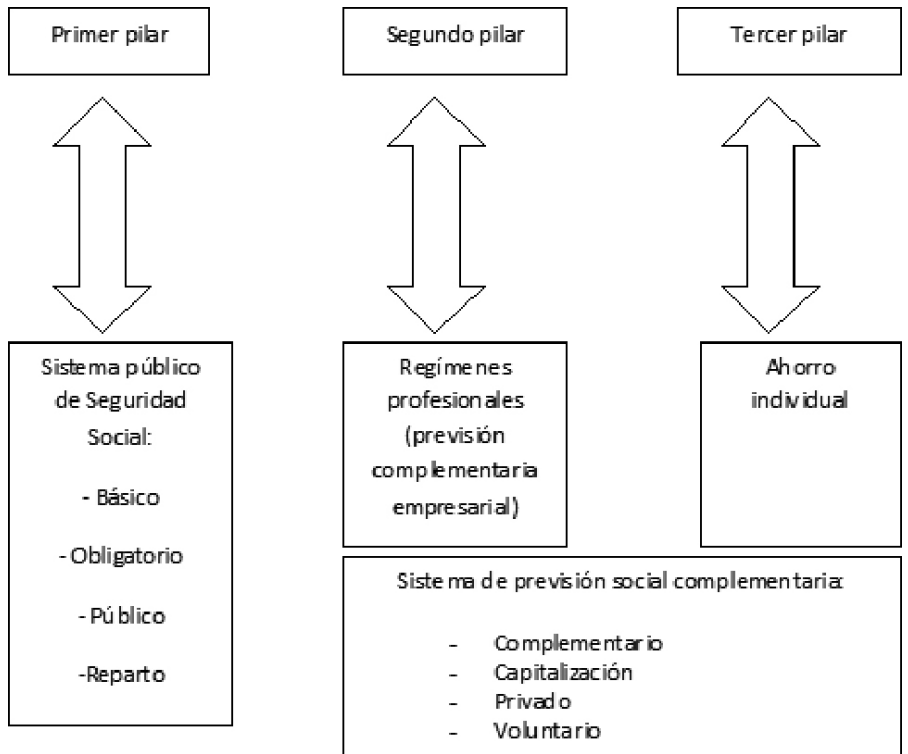

Fuente: Elaboración propia.

El primer pilar configura el Sistema de Seguridad Social público. En él se distinguen por un lado, el nivel de carácter básico, esto es, el que recoge las prestaciones no contributivas, la asistencia sanitaria, las prestaciones familiares y los servicios sociales. Todos ellos se financian mediante impuestos. Por otro lado está el denominado carácter contributivo y profesional. Este es obligatorio para todos los trabajadores/as por cuenta ajena y propia, y se financia a través de las cotizaciones tanto de los empleados como de los empresarios/as. Este pilar se basa en un sistema de reparto o "pay as you go system» que generalmente es obligatorio y se rige por los principios de solidaridad y universalidad.

El segundo pilar lo componen los regímenes profesionales, es decir, los Planes de Pensiones, Seguros Colectivos y Mutualidades que surgen del contexto de las relaciones laborales. En estos sistemas el empresario/a es el promotor de esta previsión complementaria con la participación de sus empleados/as. Este pilar puede ser obligatorio o voluntario, sustitutivo o complementario del primer pilar y además puede tener carácter público o privado. En la mayor parte de los países, este segundo pilar se financia a través de sistemas de capitalización (Alda et al., 2009). 
El tercer nivel, junto con el segundo, integran el sistema de previsión social complementaria. Este tercer pilar, se basa en el ahorro individual, es decir, se refiere a planes de pensiones, seguros y mutualidades individuales. Como resultado de ello, son justo de la decisión unilateral del individuo. Este tercer pilar tiene un carácter privado y voluntario, y existe una flexibilidad en cuanto a las aportaciones.

Los tres pilares analizados, tienen distinta repercusión o importancia en cada país, en función de un modelo de previsión social propio. De todas las formas, el denominador común es que si el primer pilar está muy desarrollado, el papel del segundo y tercer pilar será secundario, mientras que si el primer pilar es débil, los otros dos pilares ganarán fuerza. En la siguiente tabla se recoge un resumen de las características de cada uno de los pilares:

Tabla 1

Los sistemas de previsión social en diferentes países

\begin{tabular}{|c|c|c|c|c|c|c|}
\hline \multirow{2}{*}{ Estado } & \multicolumn{2}{|c|}{ Primer pilar } & \multicolumn{2}{|c|}{ Segundo pilar } & \multicolumn{2}{|c|}{ Tercer pilar } \\
\hline & Obligatorio & Voluntario & Obligatorio & Voluntario & Obligatorio & Voluntario \\
\hline Italia & $\checkmark$ & & & $\checkmark$ & & $\checkmark$ \\
\hline Portugal & $\checkmark$ & & & $\checkmark$ & & $\checkmark$ \\
\hline Alemania & $\checkmark$ & & & $\checkmark$ & & $\checkmark$ \\
\hline Francia & $\checkmark$ & & $\checkmark$ & & & $\checkmark$ \\
\hline Holanda & $\checkmark$ & & $\checkmark$ & & & $\checkmark$ \\
\hline Bélgica & $\checkmark$ & & & $\checkmark$ & & $\checkmark$ \\
\hline Finlandia & $\checkmark$ & & $\checkmark$ & & & $\checkmark$ \\
\hline Luxemburgo & $\checkmark$ & & & $\checkmark$ & & $\checkmark$ \\
\hline Reino Unido & $\checkmark$ & & & $\checkmark$ & & $\checkmark$ \\
\hline Suecia & $\checkmark$ & & & $\checkmark$ & & $\checkmark$ \\
\hline España & $\checkmark$ & & & $\checkmark$ & & $\checkmark$ \\
\hline
\end{tabular}

Fuente: Elaboración propia.

En la tabla anterior se puede observar que el primer pilar es obligatorio en todos los estados analizados y el tercer pilar, en cambio, es voluntario. A pesar de ello, las diferencias se observan en el segundo pilar donde en algunos países es obligatorio y en otros voluntario, siendo esto último lo más habitual. 


\subsection{Propuestas y tendencias recientes en el modelo de protección social}

Desde hace un tiempo atrás, y sobre todo al hilo de la crisis económica actual y los cambios demográficos, la viabilidad de las pensiones públicas viene siendo objeto de debate. La principal preocupación de la Unión Europea respecto a este tema se centra en tres aspectos ${ }^{3}$ y lo constataba de la siguiente manera la Comisión en su Comunicación de 11 de octubre de 2000 (Sáez y Sánchez): «el envejecimiento de la población y la jubilación de la generación del boom demográfico representan un reto de envergadura para este logro histórico. El envejecimiento de la población será de tal escala que, de no efectuarse reformas oportunas, podría comprometer el modelo social europeo, asi como el crecimiento económico y la estabilidad de la UE».

Además de la sostenibilidad de las pensiones, la financiación y desarrollo de la asistencia sanitaria viene siendo objeto cada vez de mayor interés. El Comité de Protección Social aprobó tres principios de carácter social, económico y de empleo, con el objetivo de garantizar la viabilidad a largo plazo de los sistemas de pensiones. En este ámbito también se estableció que cada estado miembro optara bien la opción de desarrollar y reforzar los sistemas complementarios o bien la de seguir confiando en los sistemas públicos.

De forma más reciente la Unión Europea en la COM (2007) 603 final, recogió en la Exposición de Motivos que "Los sistemas de protección social de los distintos Estados miembros deben hacer frente al problema del envejecimiento demográfico. Las reformas adoptadas o previstas en la mayoría de los Estados miembros tienden a incrementar los regimenes complementarios de pensión, algo que numerosos Estados miembros fomentan activamente». Como resultado de ello, las políticas europeas de pensiones se orientan hacia la reforma de las pensiones en cuatro direcciones: por un lado, establecer condiciones de accesibilidad más duras para adquirir una pensión pública, bajar la calidad de la prestación pública; prolongar la vida laboral y fomentar la previsión social complementaria privada (Monereo, 2013).

\subsection{El actual modelo de protección social español}

En España existe un modelo de tres pilares, en el que el primero, es decir, la Seguridad Social pública ha sido el nivel predominante. El segundo y tercer nivel han tenido escasa representación.

\footnotetext{
${ }^{3}$ Estos tres ámbitos son la llegada a la edad de jubilación de las generaciones nacidas durante el boom demográfico, las bajas tasas de natalidad y el aumento de la esperanza de vida de la población.
} 
En este tipo de sistemas la influencia de la demografía, es muy grande. En el año 1919, cuando se impuso la edad de jubilación a los 65 años, la esperanza de vida era de 45 años. Cuando en 1967 se crea el sistema que actualmente tenemos, la esperanza de vida estaba en 69 años. Por tanto, podemos observar que la edad de jubilación no ha evolucionado en la misma medida que lo ha hecho la esperanza de vida (Valero, 2014). En consecuencia, España se enfrenta al problema del impacto del envejecimiento de la población sobre el sistema de pensiones y ello requiere modificaciones en el propio sistema.

El Gobierno ha adoptado una serie de reformas en el $2011^{4}$ y el 20135. El objetivo de la reforma del 2011 fue doble: preservar el actual sistema público de reparto, blindando un sistema "mixto" con capitalización obligatoria que defendían algunos y garantizar la sostenibilidad financiera del sistema de Seguridad Social. Para ello se aumentó la edad de acceso a la jubilación ${ }^{6}$, el periodo de cómputo de la Base Reguladora ${ }^{7}$, del número de años de cotización ${ }^{8}$ y se presentó el «Factor de Sostenibilidad»". En el año 2013, se incorporaron dos mecanismos: el «Factor de sostenibilidad» y el «Índice de revalorización». En relación al «Factor de sostenibilidad» se modificaron las fechas de implantación, adelantándolo al 2019. El «Índice de Revalorización» ya está en vigor y establece la subida de las pensiones en función de la evolución de los ingresos y gastos del Sistema con un «suelo» $\mathrm{y}$ "techo» ${ }^{10}$.

Además del primer pilar, al que se le ha prestado más atención por ser el pilar más importante del estado español, no debemos olvidar que existen otros dos niveles El desarrollo de estos dos pilares en España ha sido lento e insuficiente, lo cual ha sido debido a la elevada tasa de sustitución que viene proporcionando el sistema público de pensiones, del orden del 73,9\% ${ }^{11}$. Estos pilares se materializan a través de los instrumentos de previsión social complementaria que son instrumentos de libre iniciativa, funcionamiento y gestión. Los instrumentos o

${ }^{4}$ Ley 27/2011, de 1 de agosto, sobre actualización adecuación y modernización del sistema de Seguridad Social. En el mismo preámbulo recogía lo siguiente: «El sistema de Seguridad Social tiene que seguir haciendo frente a importantes desafios, afrontando a largo plazo las exigencias derivadas, entre otras, de las tendencias de evolución demográfica, a fin de garantizar la sostenibilidad financiera de aquél».

5 La Ley 23/2013, de 23 de diciembre, reguladora del Factor de Sostenibilidad y del Índice de Revalorización del Sistema de Pensiones de la Seguridad Social.

${ }^{6} \mathrm{La}$ edad legal de jubilación pasa de los 65 a los 67 años.

7 Se amplía el período de cómputo para el cálculo de la base reguladora de los 15 a los 25 ańos.

8 Se incrementa el número de ańos de cotización exigidos para alcanzar el $100 \%$ de la base reguladora de 35 a 37 años.

9 Se aplicaría a partir del 2027, si bien actuaría cinco años después, es decir, en el año 2032.

10 Artículo 48.2.LGSS: "en ningún caso el resultado obtenido podrá dar lugar a un incremento anual de las pensiones inferior al 0,25\% ni superior a la valoración porcentual del Índice de Precios al Consumo en el periodo anual anterior a diciembre del año t, más $50 \%$.

${ }^{11}$ En la UE la media está en 53,4\%. 
modalidades que puede adoptar la previsión social voluntaria son dos principalmente: por un lado, los que se denominan Seguridad Social Voluntaria y que en nuestro ordenamiento jurídico son las mejoras voluntarias de Seguridad Social (Montoya, 1998). Por otro lado, encontramos las previsiones sociales voluntarias que están en el ámbito externo del sistema de Seguridad Social, como son por ejemplo, las Mutualidades de Previsión Social y los Planes y Fondos de Pensiones. Estos instrumentos, tienen una regulación legal específica al margen de la Ley General de la Seguridad Social (LGSS).

En nuestra opinión, dentro de los sistemas complementarios, es más eficaz y general el segundo pilar, es decir, el basado en los sistemas de empleo. Para incentivar estos sistemas algunos estados de la Unión Europea han utilizado varios instrumentos: por ejemplo, semi-obligar a la participación en estos planes. En estos casos, los trabajadores tienen la posibilidad de abandonar dicho sistema complementario, pero la tasa de renuncia es baja. Otro instrumento que podría fomentar su desarrollo es la formación e información de los ciudadanos/as. A nivel general, se tiene un conocimiento muy reducido sobre las características de los sistemas de pensiones. Adicionalmente, no se conoce el importe de la futura pensión pública y privada, y esto puede ser peligroso, lo cual incapacita la toma de decisiones sobre el consumo, ahorro y cobertura de riesgos. En el caso de España, desde la Seguridad Social únicamente se proporciona información anual sobre las cotizaciones efectuadas, con el objetivo de que si existiera un error el cotizante le pueda comunicar. Por otro lado, es posible estimar la pensión futura a través de la web de la Seguridad Social ${ }^{12}$, si bien esta aplicación está teniendo bastantes problemas técnicos. El Gobierno había preparado la denominada "carta naranja», a través de la cual la Seguridad Social iba a enviar cartas informativas por correo ordinario a los ciudadanos/as mayores de 50 ańos, informándoles sobre la cuantía estimada de la pensión que recibirán cuando se jubilen. No obstante, este envío de cartas se ha retrasado, a pesar de lo cual son muchas las entidades que ofrecen, simuladores de aportaciones para que los ciudadanos puedan planificar su jubilación privada.

Por lo tanto, se puede observar que, en España, hasta ahora ha predominado el sistema público de la Seguridad Social, es decir, el primer pilar. Sin embargo, con las últimas reformas que se han llevado a cabo para hacer frente al futuro envejecimiento de la población, se puede decir que este pilar se está debilitando. En consecuencia, al igual que otros países cercanos, España deberá buscar otras alternativas para complementar la tasa de sustitución que se perciba del sistema público, y para ello impulsar los sistemas complementarios podría ser una opción, especialmente los basados en las relaciones laborales.

12 www-seg-social.es. 


\section{Caso Maier}

\subsection{Introducción}

El Grupo Maier está integrado en Mondragón Corporación Cooperativa cuyo objeto de actividad ha evolucionado con el paso del tiempo, en función de las necesidades y oportunidades del mercado. Así, en 1975 inició su actividad en el ámbito de la matricería, inyección y cromado en el mercado del electrodoméstico. Todo ello fue posible gracias a un grupo de amigos de Gernika y el apoyo financiero de Caja Laboral Popular. En la actualidad, sus productos van orientados hacia la automoción ${ }^{13}$, donde ofrece su experiencia, buen saber hacer y compromiso con la innovación, a través de dos unidades de negocio: componentes para el exterior ${ }^{14}$ y componentes para el interior ${ }^{15}$. En cualquiera de estos dos ámbitos, el denominador común es la decoración, incluidas la pintura y el cromado, los acabados, la inyección, los ensamblajes, así como la matricería de piezas de elevada complejidad funcional ${ }^{16}$. Esto último es posible gracias al Maier Centro Tecnológico (MTC), Unidad Integral Especializada en Tecnologías $^{17}$ del plástico para el sector de automoción y en el desarrollo de decoraciones asociadas a ese material.

Maier cuenta con una presencia internacional ${ }^{18}$ a través de plantas productivas, alianzas, oficinas comerciales y centros tecnológicos con lo que pretenden

13 La orientación al sector automovilístico fue debido a la potencialidad que presentaba ese mercado así como por la oportunidad de desarrollo que la tecnología proporcionaba.

14 En este ámbito se dedican a la fabricación de la rejilla frontal, tapabujías, piezas bajo-vehículo, rejilla de entrada de aire, montantes, tapacubos, emblemas, tapas de gasolina, protectores, embellecedores del parachoques, tiradores traseros, soportes de matrículas, etc.

15 En este ámbito obtienen las bolsas de las puertas, los asaderos de puertas y techos, las rejillas de los altavoces, los cuadros de instrumentos, los embellecedores del aire acondicionado, las tapas del airbarg, etc.

16 Es precisamente en este campo dónde se contextualizan las competencias diferenciales de Maier. En el ámbito de la decoración se basan en multi-tecnologías de última generación decorativas que confieren un alto valor estético al producto. En el ámbito de la $\mathrm{I}+\mathrm{D}$, tienen establecido un "sistema de vasos comunicantes» con un centro dedicado a la innovación en nuevas estéticas y funcionales. En el ámbito de la matricería son capaces de elaborar moldes, prototipos y modelos propios y prototipos rápidos.

17 En el centro se proveen soluciones integrales en diseño, desarrollo y fabricación. Para ello se parte del estudio de los materiales lo cual permite la innovación en el ámbito de la estética y la tecnología así como de los propios materiales. También son objeto de estudio los estilos y los diseños para lo cual cuentan con programas de diseño gráfico de última generación. Se realizan simulaciones estructurales y reológicas, base de la concepción y fabricación de prototipos, moldes, útiles de montaje y se plantea el proceso de fabricación, con sus fases de inyección, acabado, decoración y montaje. Todo ello es analizado y validado minuciosamente antes de poner el proceso en marcha y obtener las primeras piezas. En bastantes ocasiones estas piezas no responden a lo esperado por lo que se realiza una supervisión de todo el proceso antes de pasar a darlo por definitivo e implantarlo en la fábrica.

18 Concretamente están presentes en Galicia, Navarra, Bizkaia, Reino Unido, Francia, Alemania, Japón, Chequia, Turquía e India. 
hacer frente a las necesidades actuales y futuras de sus clientes ${ }^{19}$. Ello le permite llevar a la práctica su estrategia global en un entorno global ${ }^{20}$. Entre los clientes de Maier se encuentran Peugeot, Citroen, Toyota, Renault, Nissan, GM, Plastic Omnium, Grupo Antolin, Daimler, Visteon, Suzuki y Faurecia. Esa orientación hacia el cliente ha sido aplaudida en el mercado con una tendencia alcista en las ventas, a pesar de la crisis actual.

La innovación, los clientes y los procesos productivos son la base del modelo de gestión de Maier. No obstante, el análisis detallado la filosofía de Maier muestra el protagonismo de un factor hasta ahora no mencionado: el/la trabajador/a. El Grupo Maier es gestionado desde una perspectiva basada en las personas y en la innovación en el camino hacia la competitividad. Así, la misión de Maier se concreta en "El Grupo Maier somos un equipo de personas en cooperación para generar riqueza en la sociedad y crear puestos de trabajo de calidad, preferentemente cooperativo, en un entorno de rentabilidad sostenible. Basamos el futuro en el desarrollo de las personas, la participación y la cooperación con otras realidades sociales, en un marco de valores compartidos. Continua afirmando que "tenemos vocación de futuro y una actitud de satisfacer, superar y anticiparnos a las expectativas del cliente, comprometidos con el entorno (medio ambiente y respeto a las características culturales de cada realidad social), la innovación y la mejora competitiva."

La visión de Maier presenta cómo quieren ser vistos en la sociedad y cuál será el papel de misma. En este ámbito se plasma también el compromiso con las personas: "Vamos a ser referente en decoración y piezas de alta complejidad funcional en el sector de automoción, diversificado con nuevos negocios y basado en personas felices de pertenecer a Maier."

En el desarrollo de su actividad en el día a día respetan una serie de valores en los que priman las personas y son los siguientes: confianza, orientación al cliente, cooperación, eficiencia y apertura al cambio.

«- Confianza:

- Actúo con sinceridad y responsabilidad.

- Comunico con claridad y transparencia y guardo confidencialidad.

- Respeto a todos y me gano el respeto de los demás.

— Orientación al cliente:

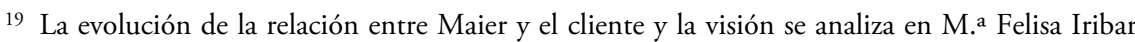
Bilbao, M. a, Larrinaga Ojanguren, M. A., (1997), Maier: Organizando el conocimiento para lograr la competitiviad, Empresas Avanzadas en Gestión, Clúster del conocimiento, ISSN: 84-605-7120-3, pág. 6 y ss.

20 Esto se recoge como "Debemos estar dónde somos estratégicos y aportamos valor para Maier en su conjunto. Una respuesta inadecuada al cliente en una ubicación afecta a Maier en su conjunto. El cliente ve a Maier como grupo y exige una respuesta como tal». 
- Reconozco y asumo que la orientación al cliente, además de un valor, es la estrategia central del Grupo Maier.

- Tengo siempre presente la importancia de satisfacer al cliente: sin él no existimos.

- Me pongo en el lugar del cliente, especialmente ante decisiones importantes y conflictos.

- Cooperación:

- Escucho y respeto las opiniones del otro.

- Priorizo lo colectivo ante lo individual.

- Participo y me implico como trabajador y socio.

- Eficiencia:

- Trabajo para conseguir resultados utilizando el mínimo de recursos.

- Me implico y cumplo los compromisos asumidos.

- Acepto que puedo fallar pero aprendo de los errores.

- Apertura al cambio:

- Acepto que las cosas cambian y me adapto a lo nuevo con actitud positiva.

- Estoy atento a las oportunidades y asumo riesgos: fomento mejoras, busco nuevas formas de hacer y genero posibilidades.

- Aprendo y comparto mis aprendizajes para innovar.»

El equipo humano de Maier Gernika a principios del 2015 lo integran 962 personas, entre socios y eventuales y se gestiona desde el departamento de Recursos Humanos. Este se divide en dos secciones: por un lado, se encuentra el/la Director/a de Gestión Socio-Laboral y por otro lado, el/la Directora/a de Desarrollo Personal que están bajo la Dirección de Planta. A su vez, en la distribución jerárquica se encuentra por encima del Director/a de la Planta, el Director/a Industrial y por último, encima de éste el Director/a General de Grupo Maier. Lo relativo a la previsión social complementaria y la Seguridad Social se gestiona desde la Gestión Socio Laboral. Bajo la Dirección de Gestión Socio-Laboral, se encuentran el/la Técnico de Recursos Humanos y los/as Administrativos/as de Recursos Humanos y el/la Recepcionista. Entre las tareas que configuran esta área están la de liderar y supervisar la administración y abono de anticipos y salarios, beneficios sociales, impuestos y cotizaciones según normativas y legislación vigente; organizar y supervisar la gestión de prestaciones de Lagun-Aro EPSV, la Seguridad Social y la gestión de jubilaciones etc.

\subsection{Previsión Social Complementaria de la Empresa Maier S.Coop}

El sistema de previsión social complementaria de Maier S.Coop diferencia entre los trabajadores/as socios/as y eventuales. Los socios/as trabajadores/ as, concretamente los de la "Tarifa 3", representan la mayor parte del perso- 
nal por lo que será en este colectivo en el que se centrará el estudio. En total son 618 personas y se pueden clasificar en dos grupos: Mano de Obra Directa (MOD) y Mano de Obra Indirecta $(\mathrm{MOI})^{21}$. El sistema de previsión de Maier se sustenta en varios vehículos: el sistema de seguridad social de los autónomos (RETA), Lagun-Aro EPSV ${ }^{22}$ y el sistema de Arogestión Ahorro-Jubilación EPSV. Como resultado de ello las cotizaciones/aportaciones de la empresa y trabajadores/as, así como las prestaciones/pensiones tienen diferentes destinos o fuentes en función de la situación laboral y edad del trabajador tal y como se describe seguidamente.

A) Sistema de la Seguridad Social: Régimen Especial de Trabajadores Autónomos (RETA).

El socio trabajador se integra en RETA $^{23}$ durante su vida laboral en Maier. La cotización al RETA es el $60 \%$ del Salario Bruto ${ }^{24}$. El trabajador voluntariamente podrá mejorar a su cargo la cotización aumentando la base de cotización dentro de los baremos establecidos por ese régimen. Durante el 2015, la horquilla está entre los 884,40 y los 3.606 euros mensuales. Esta base de cotización podrá ser mejorada por el trabajador/a dos veces al año dentro de unos límites ${ }^{25}$.

21 MOD: Es la mano consumida en las áreas que tienen una relación directa con la producción o la prestación de algún servicio. Es la generada por los obreros y operarios calificados de la empresa.

MOI: Es la mano de obra consumida en las áreas administrativas de la empresa que sirven de apoyo a la producción y al comercio.

22 Lagun-Aro EPSV, es una Entidad de Previsión Social Voluntaria que se creó el 23 de marzo de 1999. Esta Entidad tiene un carácter mutualista, proporciona el régimen de asistencia y previsión social. Entre sus principios básicos y valores destacan el mutualismo y democracia representativa, solidaridad y corresponsabilidad; competitividad en la gestión; ausencia de ánimo de lucro; adaptación permanente; y por último, transparencia. Este sistema complementario, en el caso de la empresa Maier se recoge en sus Estatutos Sociales, concretamente en el art. 28: "La cooperativa opta, a efectos de disfrute de los beneficiarios de la Seguridad Social de sus socios trabajadores, tanto consolidados como en periodo de prueba, por la modalidad de trabajadores por cuenta propia en el Régimen Especial de Trabajadores Autónomos asumiendo la Cooperativa la posición juridica de obligado ante la Seguridad y adquiriendo la condición de responsable directo y primero del pago de las cuotas. Además, "La cooperativa, asimismo, asume la obligación del pago de las aportaciones a Lagun-Aro EPSV, a beneficio de sus socioslas trabajadores/as, con objeto de financiar, en los términos regulados en los Estatutos Sociales y Reglamento de Prestaciones de Lagun-Aro EPSV, las prestaciones previstas en los mismos y, en particular, auxilio a discapacitados, asistencia sanitaria, incapacidad temporal, riesgo durante el embarazo, maternidad, ayuda al empleo, auxilio por defunción (prestaciones de reparto), jubilación, viudedad e incapacidad permanente (prestaciones de capitalización)"

23 Artículo 28 de los Estatutos Sociales.

24 Se aprobó en una Asamblea de Lagun-Aro EPSV.

25 El Régimen de Autónomos establece limitaciones para incrementar la Base de Cotización a lo largo de la vida profesional:

- Libertad de elección para menores de 47 años y aquellos con 47 años, cuya base de cotización de diciembre de 2014 haya sido igual o superior a $1.945,80$ euros/mes. 
B) Sistema de previsión social complementario empresarial: LagunAro, EPSV.

A lo largo de la vida laboral del trabajador/a, la empresa cotiza a LagunAro EPSV. Se trata de un Plan de Aportación Definida de Empleo y la cotización a este Plan es únicamente empresarial y se cuantifica a través de un «Sistema de Índices» ${ }^{26}$.

C) Sistema de previsión social complementario de empleo: Plan de Previsión Arogestion Ahorro-Jubilación Dinero y Mixto.

A través de esta tercera vía se articula la opción de que los socios/as aporten a este Plan de Previsión Arogestion Ahorro-Jubilación Dinero o al Mixto, con el objeto de mejorar la cobertura de jubilación. Se trata de un Plan de Previsión Individual de aportación definida en la que solo son admisibles las aportaciones del trabajador/a, si bien Maier aporta una cuota inicial de 50 euros al Fondo Mutual.

D) Capital social que ha generado el socio.

Cuando el socio trabajador deja de ser socio puede disponer de ese capital.

E) Ayudas económicas a las jubilaciones anticipadas.

Existe una contribución de la cooperativa que está vinculada con las jubilaciones anticipadas. Estas ayudas son también en función del ya presentado «Sistema de Índices». Así, en el 2015, para los Índices Laborales hasta 2,00 inclusive, la ayuda es de 580,20 euros/mes con un máximo de 36 mensualidades; para los comprendidos entre 2,01 y 3,00 es de 725,24 euros/mes y para superiores o iguales a 3,01 y es de 870,30 euros/mes (a partir de los 60 años y con motivo de jubilación anticipada). La duración máxima de estas ayudas es de 36 mensualidades, o hasta los 65 años del trabajador/a. ${ }^{27}$

Tras realizar un breve análisis del sistema de previsión social de Maier S.Coop. desde la perspectiva de la empresa y el trabajador/a, procederemos a continuación con el análisis de las fuentes de ingreso por pensiones, según la casuística del trabajador socio en esta empresa a partir del momento de acceso a la jubilación:

- Trabajadores autónomos que, a 1 de enero de 2015 tengan 47 años de edad y B.Cot. < $1.926,60$ euros/mes, sólo podrán incrementarla hasta 1.945,80 euros/mes.

— Mayores de 48 ańos a 1 de enero de 2015, su base de cotización mínima está comprendida entre 953,70 euros y $1.945,80$ euros/mes.

(http://www.seg-social.es/Internet_1/Trabajadores/CotizacionRecaudaci10777/Regimenes/ RegimenEspecialTrab10724/TrabAutInfoGen2k9/DebeCoti2k9Cuant/index.htm)

26 Este sistema se recoge en una tabla en la que se asocian los índices a los puestos de trabajo y se cotiza teniendo en cuenta ese nivel. De esta forma, a cada puesto de trabajo le corresponde un índice que no es posible incrementar, a no ser que se cambie de puesto.

27 Actualmente se está analizando la viabilidad del sistema a la edad de 67 ańos. 
- Trabajador de 60 a 63 ańos: el socio/a trabajador/a que cumpla los requisitos para poder jubilarse anticipadamente en Lagun-Aro EPSV ${ }^{28}$ percibirá una prestación vitalicia de Lagun-Aro EPSV de 12 mensualidades, que podrá ser mejorada por decisión unilateral del trabajador en un $125 \%, 150 \%, 175 \%$ o $200 \%$ durante el período de 60 a 63 ańos y regularizada posteriormente. Esto es lo que se denomina la jubilación redistribuida. Además, el trabajador/a percibirá la ayuda económica para las jubilaciones anticipadas de la empresa, con la que podrá seguir cotizando al RETA por convenio especial hasta cumplir la edad y los requisitos para jubilarse anticipadamente a los 63 ańos.

- Trabajador mayor de 63 años: percibiría la prestación vitalicia de LagunAro EPSV de 12 mensualidades; la prestación del RETA de 14 mensualidades en caso de cumplir los requisitos para poder jubilarse anticipadamente en el RETA; la Ayuda Económica por jubilación anticipada de parte de la Cooperativa, que variará según el Índice Laboral y también en su duración, teniendo en cuenta los meses que le quedan al trabajador para el cumplimento de los 65 ańos. Adicionalmente, a partir de esa edad tiene la opción de retirar su capital social cuando deja de trabajar, esto es, cuando deja de ser socio.

- Socio mayor de 65 años: percibirá la prestación de Lagun-Aro EPSV, que consiste en 12 mensualidades y también la prestación del RETA de 14 mensualidades. Asimismo, podría retirar el capital social acumulado.

En los tres supuestos descritos anteriormente, en caso de que el trabajador/a disponga de Arogestion Ahorro-Jubilación Dinero o Mixto, podrá retirarlo en el momento de jubilarse en forma de renta o de capital.

\subsection{Resultados de la encuesta}

Una vez presentado el sistema de previsión social de Maier, consideramos interesante analizar el grado de conocimiento y de satisfacción del personal de la empresa sobre el mismo. Para ello se confeccionó una encuesta con 17 items. Los 13 primeros analizaban el sistema de previsión social de Maier, tanto el público como el complementario. El resto de ítems, sin embargo, servían para conocer su actitud ante la futura jubilación, a través de aspectos tales como la planificación de la jubilación, la existencia de otros sistemas de previsión a parte de

28 Para poder jubilarse por Lagun-Aro EPSV, es necesario tener 60 años mínimo y deberá haber cotizado a LagunAro EPSV, como mínimo, durante ciento veinte meses, de los cuales, al menos sesenta, tendrán que corresponder al periodo inmediatamente anterior a la fecha de jubilación. 
los de Maier, así como propuestas para la empresa, si existiera un beneficio extraordinario.

La encuesta se realizó a 232 personas, lo que supone el 37,48\% del colectivo. Sobre el total de la plantilla, el 34\% fueron hombres, siendo el 23,48\% Mano de Obra Indirecta y el 42,02\% Mano de Obra Directa. Por su lado, la mujeres que participaron, supusieron el $46 \%$ mujeres de la plantilla, el $44,23 \%$ de Mano de Obra Indirecta y el 39,68\% Mano de Obra Directa de la plantilla. En términos generales la participación fue más homogénea en el colectivo de mujeres, tanto por edades como por la categoría del puesto de trabajo.

\section{Gráfico 1}

\section{Representatividad del colectivo de hombres}

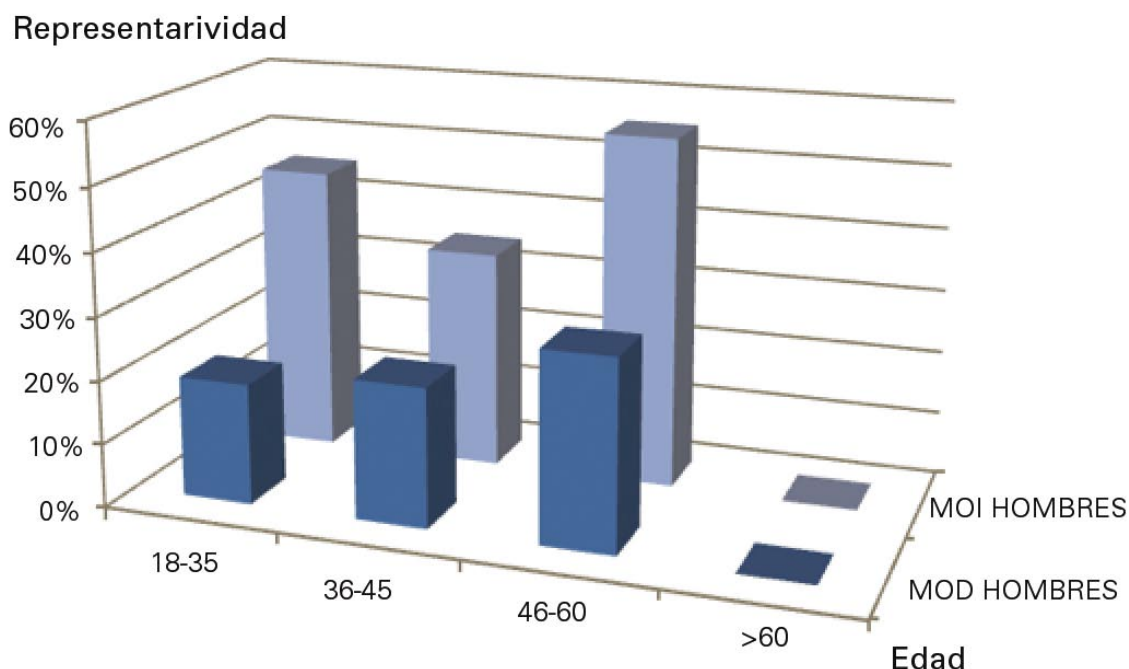

MOD HOMBRES

MOI HOMBRES 


\section{Gráfico 2}

Representatividad del colectivo de mujeres

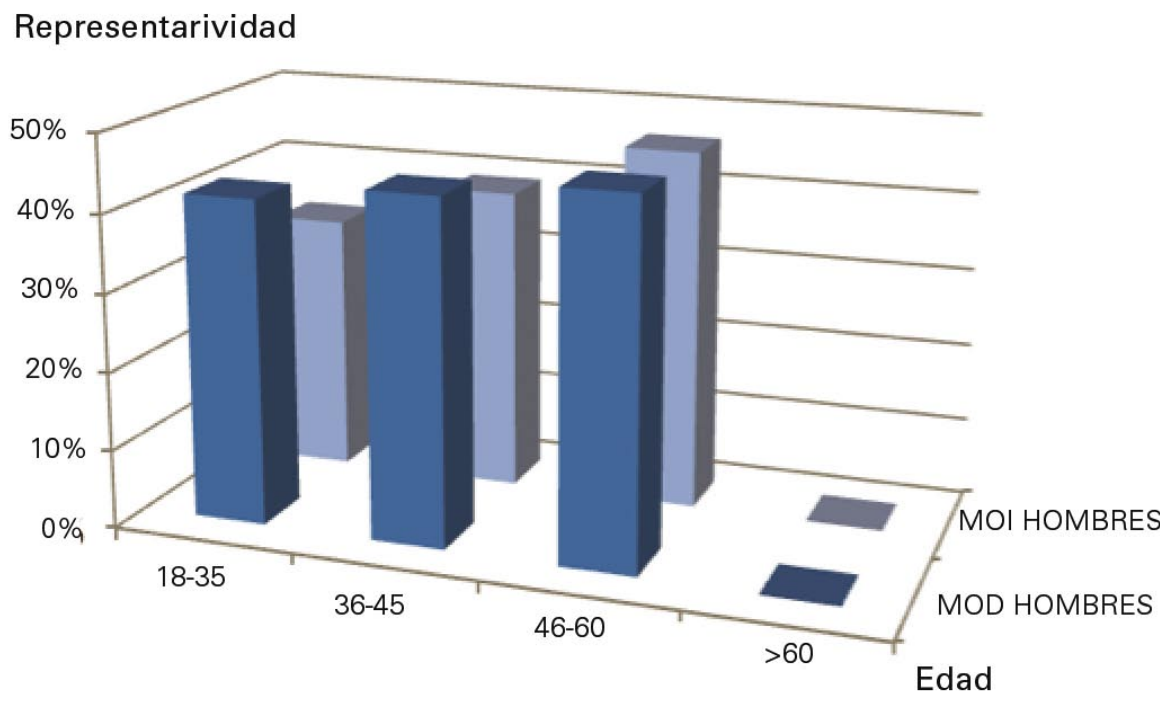

MOD HOMBRES MOI HOMBRES

El grado de conocimiento del sistema de pensiones de Maier fue consultado a través de diferentes cuestiones. El mayor conocimiento del sistema no se concentra en un colectivo concreto, esto es, varía en función del sexo y la categoría profesional. En relación al sistema al que se cotiza, las respuestas mayoritarias fueron acertadas, independientemente del sexo, edad y categoría profesional. Esto mismo ocurre también en los ítems relativos a las cotizaciones. Sin embargo, en esta ocasión tan sólo el 35\% como media es capaz de cuantificar a ciencia cierta el importe de la cotización suya, así como la que la empresa realiza a LagunAro. Otra variable clave en el estudio fue la posibilidad de aumentar la cotización. En este caso, el colectivo de mujeres y hombres de Mano de Obra Indirecta optaron mayoritariamente, un $80 \%$ y un $57 \%$ respectivamente por la respuesta correcta. En el colectivo Mano de Obra Directa, la respuesta media acertada alcanzó el $54 \%$ tanto de los hombres como mujeres de los casos. No obstante, la pregunta que peores resultados ha obtenido, a gran distancia del resto de preguntas, ha sido la relativa a la edad de acceso a la jubilación: el colectivo de hombres ha respondido mejor que el de mujeres, pero en niveles de acierto de entre el $2 \%$ y el 17,8\%. El acceso a la prestación de LagunAro también suscita dudas entre el personal, especialmente entre puestos de Mano de Obra Directa. En esta cuestión, el nivel de aciertos se 
situó entre el 44\% de las mujeres de Mano de Obra Indirecta y el $26 \%$ de las mujeres de Mano de Obra Directa. Del total de los encuestados, algo menos de la mitad conoce el mecanismo por el que se pueden incrementar las cotizaciones a Lagun Aro, si bien la mayoría de ellos identifica el sistema que se utiliza para valorar el importe de la cotización, esto es, el «Sistema de Índices». El colectivo de Mano de Obra Directa, hombres y mujeres, lideran las respuestas acertadas sobre la ayuda económica a la jubilación anticipada, si bien en todos los colectivos más del $50 \%$ lo conocen. La distribución de las respuestas se recoge en el siguiente gráfico.

Gráfico 3

Conocimiento del sistema de previsión social de Maier

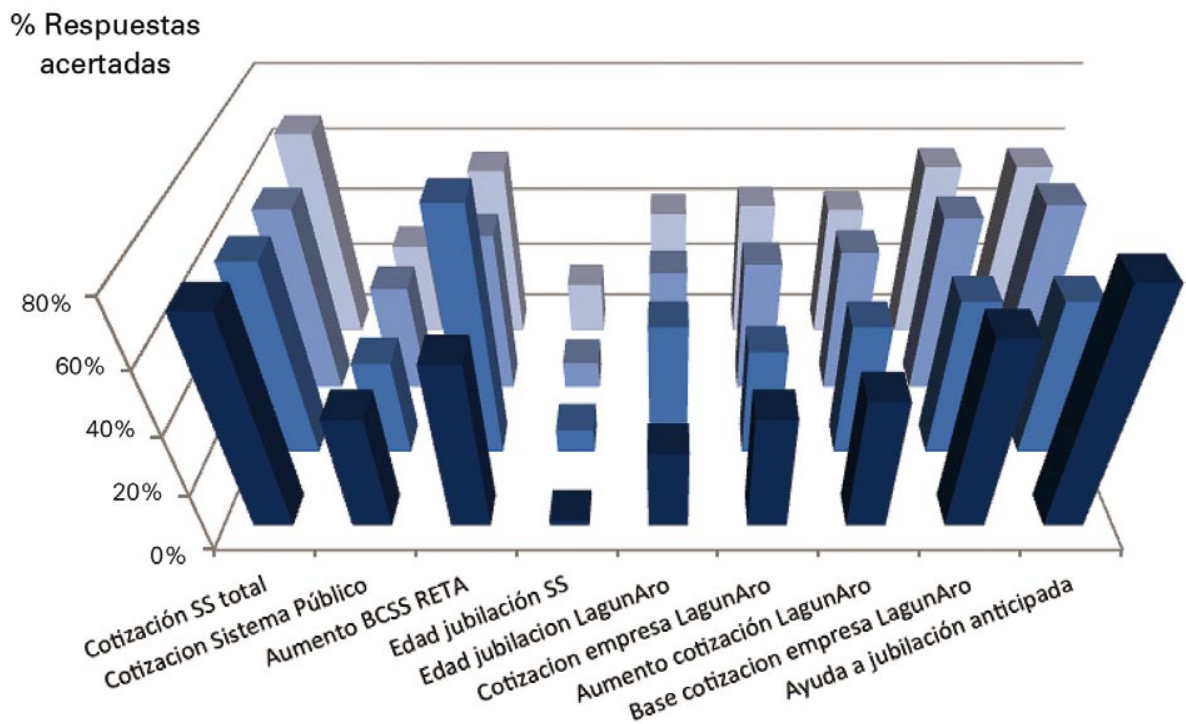

MOD MUJERES MOI MUJERES WOMBREMOD HOMBRE MOI

El resto de ítems de la encuesta indagan sobre aspectos relacionados con la previsión a nivel individual así como por la preocupación sobre la futura jubilación. Así, el personal encuestado mayoritariamente no tiene contratado un sistema complementario de previsión social y esto es especialmente notorio entre el colectivo de Mano de Obra Directa, sean hombres o mujeres. Entre el colectivo de mujeres de Mano de Obra Indirecta la previsión se orienta hacia una EPSV más que hacia un Plan de Pensiones, al contrario de lo que ocurre en el colectivo de hombres, que tienen más Planes de Pensiones. En cualquiera de los casos, es especialmente notorio la baja acogida del sistema 
implantado por la empresa, Arogestión EPSV, ya que solo lo tienen contratado sobre el $5 \%$, salvo en el colectivo de hombres de Mano de Obra Indirecta, que es el 10,13\%. El detalle de estos resultados se puede constatar en el siguiente gráfico.

\section{Gráfico 4}

Los sistemas complementarios de previsión social del personal de Maier

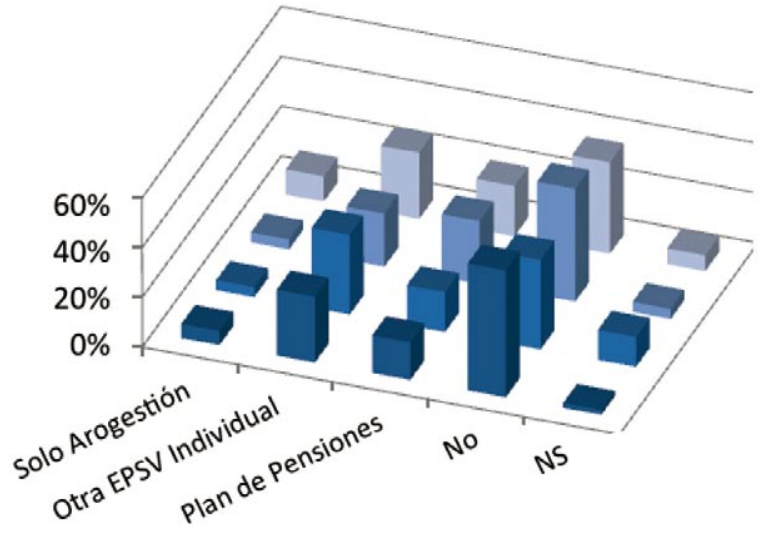

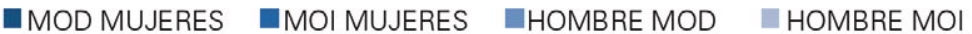

El escaso éxito de Arogestión EPSV entre el personal encuestado puede ser debido a diversas causas. Entre las que habitualmente justifican ese comportamiento están el que la política de inversión sea prudente, la falta de información o de rentabilidad, comisiones altas u otro tipo de motivos. El resultado de este ítem es claro: los encuestados no destinan su ahorro particular a Arogestión EPSV especialmente por la falta de información. También se apuntan otras razones para la no inversión, entre ellas, las 2 razones más populares han sido, el ser demasiado joven para ello y el necesitar el dinero para los gastos del día a día. 


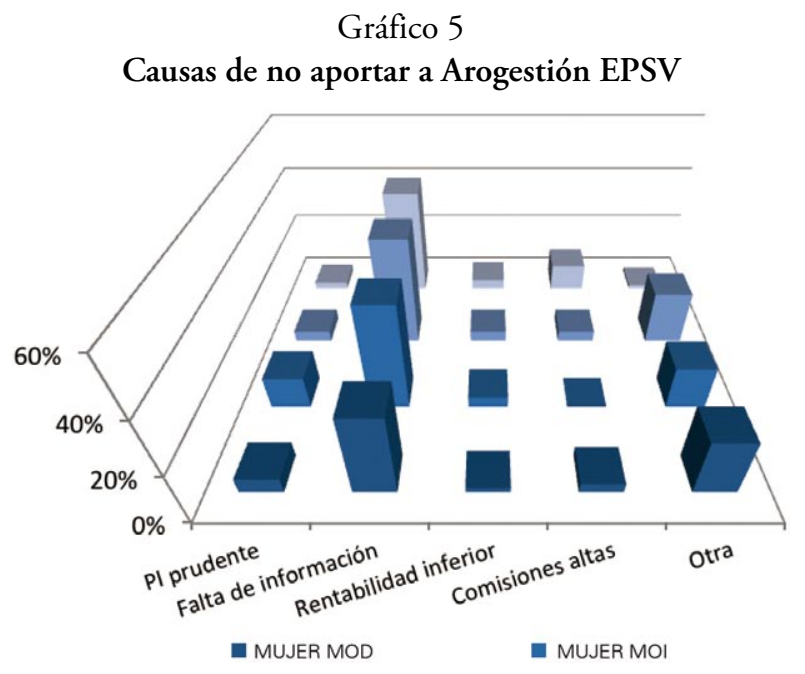

La planificación de la jubilación es un ejercicio que puesto en marcha desde bien temprano permite asegurar unos ingresos a la jubilación que permitan mantener el poder adquisitivo previo a la jubilación. Las respuestas del ítem que analiza este apartado ilustran que la mayoría del personal no tiene planificado a la fecha de hoy la jubilación. Otros, en cambio si lo hacen. Entre ellos, la mayoría lo hace en colaboración con la empresa, siendo el colectivo de hombres de Mano de Obra Indirecta y Mujeres de Mano de Obra Directa, con un $40 \%$ y un 30\% los más asiduos. El resto del colectivo este resultado se sitúa sobre el $25 \%$.

\section{Gráfico 6}

\section{Causas de no aportar a Arogestión EPSV}

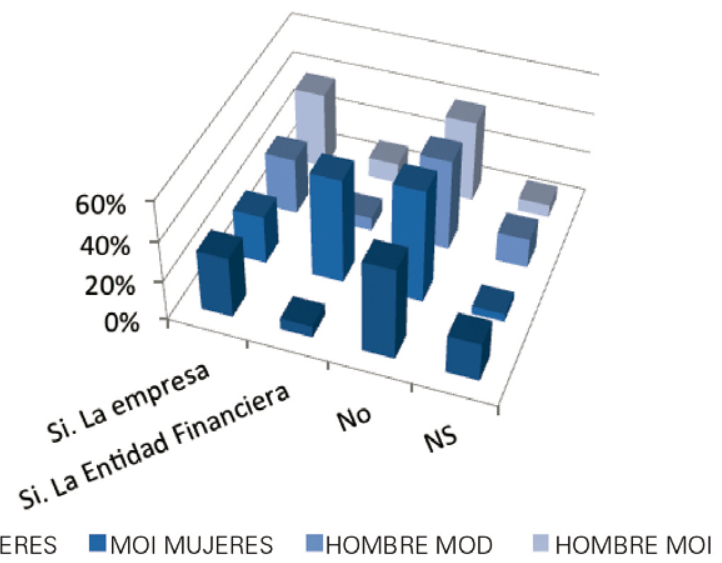


Finalmente se preguntó al personal sobre el destino de futuros e hipotéticos beneficios extraordinarios en Maier. La respuesta por la que se ha optado mayoritariamente ha sido la $\mathrm{I}+\mathrm{d}+\mathrm{i}$, colocándose en segunda posición la mejora del salario. La opción vinculada con la previsión social se coloca en tercera posición, casi a la par de otras opciones, entre las que destacan mayores facilidades para la conciliación de la vida familar y laboral y subvenciones para el comedor.

\section{Gráfico 7}

\section{Destino posible beneficio extraordinario de Maier}

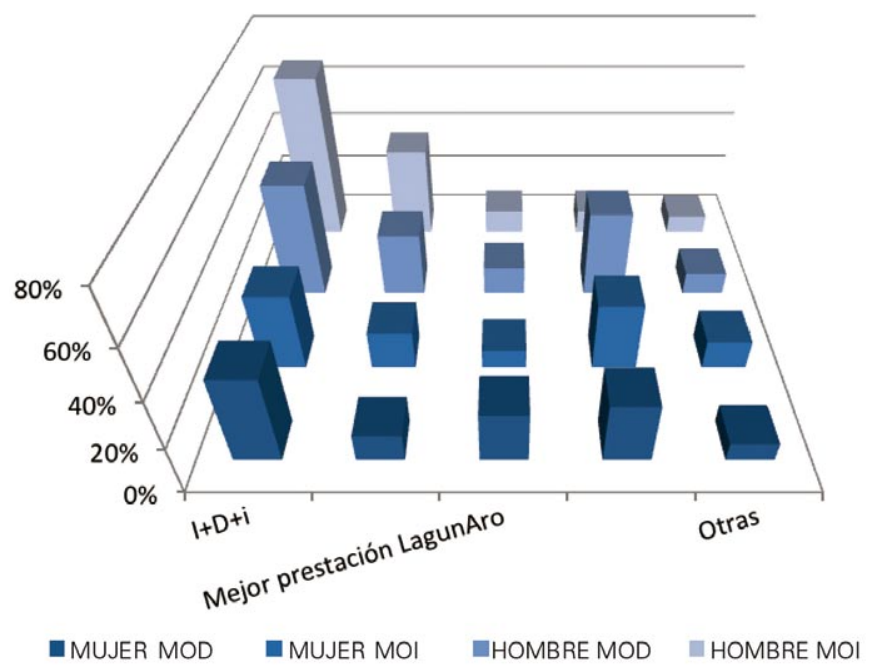

\section{Conclusiones}

Una vez realizado el análisis teórico de la cuestión y teniendo en cuenta los resultados extraídos de las encuestas, destacamos diez conclusiones, de las cuales, cinco son relativas al marco teórico y otras tantas al caso de Maier.

Como primera conclusión se puede destacar la indefinición del concepto de la protección social en la Constitución de 1978. En consecuencia, han sido muchos autores los que se han pronunciado sobre tal tema, con distintos criterios de clasificación. Tras analizar la doctrina, se puede concluir que la protección social no sólo es Seguridad Social y la Asistencia Social, sino también entran las ayudas a la vivienda, promoción del empleo y las ayudas para hacer frente a las consecuencias de catástrofes naturales.

En segundo lugar, en términos generales, el sistema global de pensiones se estructura en tres niveles. Estos pilares tienen distinta importancia en cada país, 
ya que varía según el modelo de previsión social adoptado, esto es, en el caso que el primer pilar éste muy desarrollado, el papel de los otros dos pilares será secundario y viceversa.

En tercer lugar, tanto las recomendaciones de la OCDE como las de la UE, van encaminadas en la misma dirección, esto es, en el impulso a la protección social complementaria.

En cuarto lugar, si nos centramos en el caso concreto de España, el Gobierno está realizando continuas reformas para hacer frente al problema del envejecimiento de la población.

En quinto lugar, podemos destacar que la proximidad a la edad de jubilación ha sido un elemento clave en el nivel de respuestas. La encuesta contó con un mayor nivel de respuesta entre el colectivo de mujeres, especialmente de aquellas con una edad comprendida entre los 46 y 60 años. Esta edad también registra niveles de participación altos entre el colectivo de hombres, especialmente los del colectivo de Mano de Obra Indirecta. En sexto lugar, los resultados de la encuesta realizada al personal de Maier sobre la previsión social en general y de Maier en particular, apunta que éste es bien conocido por el personal en algunos aspectos como, por ejemplo, el Régimen de la Seguridad Social al que cotizan y su importe, lo que Maier cotiza por ellos a LagunAro y la existencia de la ayuda a la jubilación anticipada de Maier.

En séptimo lugar destacar que otros aspectos, especialmente la edad de acceso a la jubilación tanto a efectos de la Seguridad Social como de LagunAro, así como el aumento de la cotización a LagunAro y la cotización al sistema público, suscitan dudas.

En octavo lugar, apuntar que el sistema de previsión social Arogestión no cuenta con gran popularidad entre el personal, debido principalmente a la falta de información. Además la mayoría no tienen otro sistema complementario de previsión social. En noveno lugar, resaltar que la mayor parte de las respuestas recogían la ausencia de acciones en el ámbito de la planificación de la jubilación y los que lo tenían lo hacían principalmente a través del área de recursos humanos de Maier.

En último lugar, pero no por ello menos importante, resaltar que el personal de la empresa está implicado en la actividad de Maier, tal y como se desprende del hecho de que el personal mayoritariamente desearía que, en caso existir un beneficio extraordinario en Maier, éste se destinara a la investigación, el desarrollo y la innovación. La segunda opción más aplaudida sería un mayor salario.

Para finalizar, en nuestra opinión el impulso a la previsión social complementaria debe venir por la vía de las Relaciones Laborales, como se ha realizado en la empresa analizada. 
Tal y como se recogió en la presentación de Maier, en el desarrollo de su actividad integran valores como la confianza, orientación al cliente, cooperación, eficiencia y la apertura al cambio. En ese camino queremos hacer llegar a la empresa las siguientes propuestas:

- Acciones formativas sobre las cotizaciones a la jubilación, del empleado y la empresa, y su efecto sobre la futura pensión de jubilación para todo el colectivo en general y especialmente para el de Mano de Obra Directa.

- Formación destinada a todo el colectivo sobre la edad de acceso a la jubilación.

- Habilitar y/o potenciar los canales de información sobre el sistema Arogestión EPSV.

- Implantar acciones encaminadas a fomentar la planificación de la jubilación en colaboración con la empresa y Arogestión EPSV. Lo ideal sería hacerlo desde que se consolida la relación laboral con Maier y revisarlo anualmente.

- Aplaudir el compromiso del personal de la empresa por su decidido apoyo a que en Maier se fomentara la Investigación, Desarrollo e Innovación, si hubiera beneficios extraordinarios.

- Felicitar a la dirección y agentes sociales de la empresa por el modelo de previsión social de Maier, completo y flexible, que sin lugar a dudas contribuye y contribuirá a que el personal de Maier sea más feliz. Todo ello es una evidencia de la Visión de Maier: «Vamos a ser referente en decoración y piezas de alta complejidad funcional en el sector de automoción, diversificado con nuevos negocios y basado en personas felices de pertenecer a Maier.»

\section{Bibliografía}

Alarcón Caracuel, M.R. (1996), «Hacia el derecho de la protección social», en López López, J., Seguridad Social y Protección social: temas de actualidad, Madrid, Ed. Marcial Pons.

Alda García, M., Ferruz Agudo, L., Rivas Compains, J. (2009) Los sistemas de previsión social en la Unión Europea. Los sistemas públicos y privados de pensiones, Madrid, Quiasmo Editorial.

López Cumbre, L. (2011), «Protección social y comunidades autónomas», en. Los nuevos marcos de relaciones laborales en el renovado estado de las Autonomías. XXI Congreso $\mathrm{Na}$ cional de Derecho del Trabajo y de la Seguridad Social, Valencia, Ed. Tirant lo Blanch.

Monereo, J.L. (2013), Gestión pública y gestión privada de las pensiones, Madrid, X Congreso Nacional de la Asociación Española de Salud y Seguridad Social.

Montoya Melgar, A. et.al. (1998) Curso de Seguridad Social, Madrid, Universidad Complutense. 
Sáez Fernández, F.J., Sánchez Martínez, M.T., Las mutualidades de previsión social y los sistemas de protección complementarios, Universidad de Granada, Fundación Once, Documento de Trabajo n. ${ }^{\circ} 3$.

Terradillos Ormaetxea, E., «La regulación del factor de sostenibilidad y del índice de revaloración del sistema de pensiones de la Seguridad Social a la luz de la entrada en vigor del protocolo facultativo del Pacto Internacional de Derechos Económicos, Sociales y Culturales", Revista General del Derecho del Trabajo y de la Seguridad Social, n. ${ }^{\circ} 36,2014$.

Valero, D. (2014), «Perspectivas del sistema público de pensiones y el papel de la previsión complementaria" en. La previsión social complementaria. Papel y claves de desarrollo, Vitoria-Gasteiz, Revista Vasca de Economía, Ekonomiaz, n. 85.

\section{Webgrafía}

Sistema europeo de Estadísticas Integradas de Protección Social, http:/europa. eu/legislation_summaries/employment_and_social_policy/situation_in_europe/ c10141_es.htm (Fecha de consulta: 21/05/2015)

SEguridad Social http://www.seg-social.es/Internet_1/index.htm (Fecha de consulta: 22/05/2015)

Seguridad Social-Trabajadores, http://www.seg-social.es/Internet_1/Trabajadores/ CotizacionRecaudaci10777/Regimenes/RegimenEspecialTrab10724/TrabAut InfoGen2k9/DebeCoti2k9Cuant/index.htm (Fecha de consulta: 21/05/2015)

\section{Tablas, figuras y gráficos}

FIGURA 1. Sistema de protección social.

TABLA 1: Los sistemas de previsión social en diferentes países.

Gráfico 1: Representatividad del colectivo de hombres.

GráfICO 2: Representatividad del colectivo de mujeres.

Gráfico 3: Conocimiento del sistema de previsión social de Maier.

Gráfico 4: Los sistemas complementarios de previsión social del personal de Maier.

Gráfico 5: Causas de no aportar a Arogestión EPSV.

Gráfico 6: Causas de no aportar a Arogestión EPSV.

GráfICo 7: Destino posible beneficio extraordinario de Maier. 